\title{
Hombres que tienen sexo con hombres (HSH): reflexiones para la prevención y promoción de la salud*
}

\section{Men that have sex with men (MSM): reflections for prevention and health promotion}

\section{Homens que têm sexo com homens (HSH): reflexões para a prevenção e promoção da saúde}

Fecha de recepción: 10-11-13 Fecha de aceptación: 17-03-14

doi:10.11144/Javeriana.RGYPS13-26.htsh

John Harold Estrada-Montoya**

Este documento constituye un ensayo el cual presenta la opinión sustentada del autor como resultado de muchos años de investigación.

Profesor titular, Facultad de Odontología, Universidad Nacional de Colombia. Doctor en Salud Pública de la misma institución.Correos electrónicos: jhestradam@unal.edu.co,jhestradam@gmail.com. Dirección de correspondencia: Avenida Caracas No. 53-46, apartamento 401. Bogotá, Colombia. 


\section{Resumen}

Este artículo parte de la evidencia sobre la reemergencia, a nivel mundial, de la infección por VIH en HSH. Según ONUSIDA, Latinoamérica aporta el mayor número de infecciones nuevas de VIH en HSH, después de los Estados Unidos de América. Asimismo, el 35\% de las personas que viven con VIH en Latinoamérica son HSH. Además, reflexiona sobre las conductas respecto al sexo no protegido, construidas sobre creencias o supuestos personales basados en: intuición, confianza y emociones. Las relaciones sexuales no protegidas en HSH podrían convertirse, inconscientemente, en un acto simbólico de rebelión y transgresión placentero. Se concluye que las necesidades no sexuales de los HSH, que los llevan a tener sexo no protegido, comprenden varias situaciones, sensaciones y sentimientos. Los programas de prevención deben incluir y enfatizar en los múltiples procesos que intervienen en la toma de decisiones y aceptar las situaciones de vulnerabilidad esta población.

Palabras clave: VIH; identidad de género; homosexualidad; autocuidado; conducta sexual; vulnerabilidad social

Palabras clave descriptor: VIH; identidad sexual; homosexualidad; autocuidado; conducta sexual; vulnerabilidad social

\section{Abstract}

This paper begins whit the evidence about the re-emergence, worldwide, of HIV infection in MSM. According to UNAIDS, Latin America contributed the largest number of new HIV infections in MSM, after the United States of America. Also, 35\% of people living with HIV in Latin America are MSM. In addition, to reflect on the behaviours front the unprotected sex, built on beliefs or personal assumptions based on: intuition, trust and emotions. The unprotected sex in MSM could become, unconsciously, in a symbolic act of rebellion and transgression pleasurable. It concludes that nonsexual needs of MSM, that lead them to have unprotected sex, comprise several situations, sensations and feelings. The prevention programs must include and emphasize in the multiple processes involved in decision making, and accepting the vulnerabilities of MSM.

Keywords: HIv; gender identity; homosexuality; self-care; sexual behaviour; social vulnerability Keywords plus: HIv; gender identity; homosexuality; self-care; sexual behavior; social vulnerability

\section{Resumo}

Este artigo é baseado na evidência sobre o ressurgimento, em todo o mundo, de infecção pelo HIV em HSH. Segundo UNAIDS, América Latina fornece o maior número de novas infecções pelo HIV em HSH, depois dos Estados Unidos da América. Também, 35\% das pessoas que vivem com HIV na América Latina, são HSH. Ademais, reflexão sobre o comportamento contra o sexo desprotegido, construídos sobre crenças pessoais e suposições baseadas em: intuição, confiança e emoções. O sexo desprotegido entre HSH poderia tornar-se, inconscientemente, em um ato simbólico de rebeldia e transgressão prazerosa. Conclui-se que as necessidades não-sexuais de MSM, que levá-los a ter relações sexuais desprotegidas, compreendem diversas situações, sensações e sentimentos. Os programas de prevenção devem incluir e enfatizar em os vários processos envolvidos na tomada de decisão, e aceitar as vulnerabilidades dos HSH.

Palavras chave: HIV; identidade de gênero; homossexualidade; autocuidado; comportamento sexual; vulnerabilidade social

Palavras chave descritores: HIv; identidade de gênero; homossexualidade; autocuidado; comportamento sexual; vulnerabilidade social 


\section{Introducción}

La categoría hombres que tienen sexo con hombres (HSH) nació a mediados de la década de los noventa, en vista de la necesidad de priorizar fondos en poblaciones de alta vulnerabilidad, sin insultar su identidad, por iniciativa del Centro de Control de Enfermedades de Atlanta. El término se empezó a utilizar en la literatura científica producida en inglés, a partir de 1997, bajo la sigla MSM (men who have sex with men). Debido a varios factores, incluyendo aspectos políticos que pretendían mantener enmascarada a esta subpoblación, en Latinoamérica la categoría HSH se empezó a difundir en estudios y publicaciones científicas solo hasta finales de la década de los noventa. A partir del año 2000 son variadas y numerosas las fuentes que se pueden encontrar que utilizan esta categoría, aunque destacan dos pioneras con valiosos aportes y rutas teóricas (1-6).

Más adelante, empezó a ganar posicionamiento el discurso "El sIDA es problema de todos", que si bien aparece con una intencionalidad incluyente, trajo aparejados problemas como que se dejaron de hacer intervenciones centralizadas en grupos específicos y por este motivo muchas personas empezaron a considerar el VIH como problema ajeno o de "otros", o de "todos" y a la vez de "nadie". Las políticas implementadas bajo esta premisa podrían ser calificadas de heterocentradas y adultocéntricas (pensadas para “todos” y “todas”), lo cual dejó por fuera a numerosos hombres de diferentes edades y orientaciones sexuales. A partir de este momento, la infección del viH/sidA, que traía cifras alarmantes, continuó devastando a los grupos de HSH, pero la falta y/o la inoperancia de las acciones gubernamentales, así como la invisibilización de esta población, han permitido que los indicadores epidemio-

46 lógicos se disparen en toda América Latina y el Caribe hasta valores de prevalencia de infección VIH entre el 5 y el $40 \%$ en HSH en algunas ciudades de la región (6-13).

En el planeta, al revisar las nuevas infecciones por vía de transmisión, el $75 \%$ se producen entre hombres heterosexuales, y en nuestro continente se presentan simultáneamente aumentos en las tasas de incidencia en hombres homosexuales y otros HSH y heterosexuales, pero dado el menor tamaño poblacional de la comunidad de $\mathrm{HSH}$ respecto a la de hombres heterosexuales, las repercusiones en este grupo son de mayor proporción e impacto y requieren acciones inmediatas y contundentes $(11,14)$.

Las intervenciones realizadas con grupos de HSH en los años ochenta resultaron en reducciones significativas en las prácticas de riesgo y en la prevención de nuevas infecciones entre estos, pero en los años noventa y en los primeros años del siglo XXI la evidencia disponible indica que la infección por el VIH está reemergiendo de manera alarmante entre los HSH en todo el mundo (desarrollado y subdesarrollado), incluso en regiones donde no se le consideraba ni siquiera posible, como África subsahariana, China y los países del mediterráneo.

La población de HSH se ha convertido en un factor crucial que aporta la actual configuración a la epidemia, junto con otras poblaciones vulnerables como los/las usuarios (as) de drogas endovenosas (UDE), las mujeres transgeneristas y los/las trabajadores (as) sexuales (TS). Por este motivo se hace indispensable profundizar mediante estudios cualitativos en el conocimiento de los aspectos estructurales que condicionan su mayor vulnerabilidad a la epidemia.

Para dar respuesta a esta necesidad, la mayor parte de los estudios de prevalencia realizados con poblaciones vulnerables, ocultas o con prácticas estigmatizadas y por ello de 
difícil acceso a los investigadores, se han realizado siguiendo metodologías de muestreo que a pesar de pretender ser aleatorias, terminan convirtiéndose en intencionales, no probabilísticas o de conveniencia, lo cual no ha permitido hasta ahora establecer con precisión si la cifra de prevalencia informada por muchos de los estudios disponibles para examen está sobrerrepresentada o subrepresentada en relación con el comportamiento real de la epidemia en este subgrupo $(15,16)$.

Para acceder a poblaciones con estas características, se han propuesto varios métodos de investigación, pero todos han presentado dificultades prácticas asociadas a la condición propia de los grupos de difícil identificación y todas presentaban dos sesgos comunes: no lograban una muestra inicial aleatoria y los resultados no representaban totalmente a la subpoblación estudiada.

Para intentar superar estos inconvenientes y a la vez resolver la pregunta fundamental, Broadhead y Heckathorn (17) describieron y aplicaron un nuevo método en un estudio de prevención de VIH en usuarios de drogas intravenosas en Connecticut. Este nuevo planteamiento metodológico fue presentado por Heckathorn (15), con el nombre de muestreo dirigido por entrevistados, o RDs (por sus iniciales en inglés Respondent Driven Sampling). Este método asume que los mejores individuos para acceder a una población oculta son los que pertenecen a la población que se piensa acceder. Estas primeras personas que son contactadas para iniciar el estudio se conocen con el nombre de semillas, quienes tienen el reto de contactar personas conocidas dentro de sus redes sociales para que a su vez ingresen al estudio. El método RDS plantea una aproximación muestral tipo cadena de referencia que además incorpora las teorías de redes sociales para obtener un tamaño de la muestra representativo de la población objeto de estudio y para reducir el sesgo ya mencionado $(15,18-20)$.

La ventaja más importante que presenta este método es que puede acceder a individuos que no acuden a centros de apoyo institucional, clínicas o espacios de encuentro social y a personas de estratos socioeconómicos bajos, lo cual cobra importancia al observar que la mayoría de casos de VIH/SIDA en población HSH se reportan en el grupo más afectado por la pobreza. El reclutamiento en cadena permite que al expandirse a diferentes niveles y dentro de redes diferentes, la muestra se componga de un grupo poblacional grande y variado y que pueda considerarse muestreo aleatorio, con las implicaciones de inferencia que esto trae aparejado (21-24).

Los datos epidemiológicos disponibles hasta hoy permiten afirmar que en muchos de los países de la región latinoamericana (y Colombia no es la excepción) la epidemia se encuentra concentrada en poblaciones de HSH. En varias de estas las cifras van en aumento desproporcionado en relación con las cifras epidemiológicas del país respectivo. Según los reportes de onUsida de los últimos años, América Latina aporta el mayor número de infecciones nuevas de VIH en HSH después de los Estados Unidos de América.

De los estudios de prevalencia e incidencia entre HSH disponibles en América Latina y el Caribe, en los cuales se realizó comparación y desagregación por categorías de transmisión, entre ellas la sexual, puede derivarse que el porcentaje de personas viviendo con VIH que son HSH, en comparación con el total de la población afectada, oscila en valores que van del 10 al 15\% en países del Caribe no latino, Caribe latino y Centroamérica; 30 a $35 \%$ en el Brasil y el Cono Sur; hasta un $50 \%$ en el área andina y un $60 \%$ en México, con lo cual el promedio para la región estaría cercano al $35 \%$, cifra elevadísima si se considera el 
tamaño relativamente pequeño de la población de HSH en relación con la población heterosexual en dichos países. Los estudios de estimación del tamaño de la población HSH para América Latina, aunque incipientes, muestran valores que oscilan entre $6 \%$ y máximo $20 \%(6,14,25-27)$.

El hecho de que durante años se asumiera una postura de cierta aceptación natural con respecto a la mayor afección de grupos de HSH, sumado a la situación de estigma y discriminación que experimentan estos hombres en la mayor parte del mundo, podría explicar en parte por qué pese al paso del tiempo y a los esfuerzos de organizaciones no gubernamentales (ONG) y otros colectivos que agrupan hombres gay o de diversas orientaciones sexuales, no se ha logrado controlar la infección y, por el contrario, pareciera que este objetivo se encuentra muy lejos de lograrse y cuestiona seriamente los esquemas y modelos de prevención utilizados hasta el momento (28-30).

En muchas ocasiones por no realizar adecuadas historias clínicas con los pacientes, o por el estigma asociado a las prácticas homosexuales, se presume una transmisión de tipo heterosexual, y esto contribuye al subregistro y a invisibilizar el peso de la epidemia en este grupo. Esta situación es crucial al enfrentar los desafíos del diseño de programas de prevención, toda vez que se encuentra arraigada una idea de juventud como periodo eminentemente heterosexual, lo que deja por fuera de las acciones educativas y de prevención a los jóvenes HsH $(31,32)$.

Desde la nueva lógica que opera para la vigilancia epidemiológica de segunda generación (en particular los estudios de comportamiento), se hace necesario estimar de la manera más precisa posible el tamaño real de la población de HSH (14); sin embargo, no existe un método exacto y estandarizado que guíe el proceso de estimación de poblaciones vulnerables al viH. La estimación de tamaños poblacionales tiene como fin último alertar a los diseñadores de políticas públicas acerca de la existencia y la magnitud de los grupos poblacionales con alta vulnerabilidad al VIH, para facilitar la planeación y la distribución de servicios adecuados; no obstante, es importante recordar que la estimación poblacional no puede ser la respuesta a los problemas específicos de cada grupo ya que no resuelve los inconvenientes ni los posibles errores de planeación y programación $(31,33)$.

El grupo de HSH está muy descuidado en varias regiones del mundo, incluida Latinoamérica. Son hombres muy difíciles de identificar y en la mayoría de los casos el tamaño poblacional es excepcionalmente difícil de estimar. Aunque en la mayoría de países, al menos una porción de la población de HSH es abiertamente gay y se puede encontrar en bares y sitios de encuentro identificados como de "socialización gay", se debe tener en cuenta que según estimativos, entre el 40 y el $45 \%$ de la población de HSH acude a estos sitios de encuentro, en donde se han enfocado la mayoría de las campañas, lo cual de entrada deja por fuera más de la mitad de la población objeto de estas acciones (34).

Se requiere, por consiguiente, continuar con estudios de seroprevalencia en grupos clave; profundizar en la implementación de metodologías cualitativas con protagonistas destacados de estos grupos; caracterizar la variedad y diversidad de conductas y comportamientos sexuales asociadas a esta población; estimar la frecuencia y calidad de la utilización de preservativos y evaluar el impacto comportamental inmediato y al largo plazo de las estrategias educativas y de prevención orientadas a esta población, con un imperativo ético primordial: la protección de los derechos humanos y la atención a HSH 
particularmente frágiles, como los menores de edad, los prisioneros, los trabajadores sexuales, los migrantes y los usuarios de drogas endovenosas.

La pandemia de VIH en el grupo de HSH puede calificarse de devastadora, considerando que este continúa siendo el grupo en situación de mayor vulnerabilidad y por ende requiere una respuesta social clara, efectiva y bien establecida. No hacerlo puede tener como efecto la sinergia de la epidemia de este grupo con las epidemias de VIH existentes en todo el mundo, como la asociada a las compañeras sexuales de algunos HSH y a sus hijos (10-14,35,36).

Dado que las relaciones sexuales entre hombres son una realidad en nuestra sociedad y que las prácticas homosexuales se pueden experimentar entre dos homosexuales, entre un homosexual y un heterosexual, entre un bisexual y un homosexual, entre hombres transgeneristas y entre dos heterosexuales, se sugiere entonces hablar de una homosexualidad permanente y de una homosexualidad circunstancial, y se prefiere por ahora utilizar el término (cada vez más restringido y en vías de superación), de hombres que tienen sexo con hombres (HSH), dado que este agrupa comportamientos y no identidades de género. Lo que es más difícil de enfrentar es que en muchos casos y países en particular, estas relaciones se pueden dar o no con el reconocimiento de una identidad homoerótica por parte de los participantes, lo cual genera una primera ruptura a la conceptualización de HSH y, por ende, a las estrategias de intervención con ellos (37).

A este respecto es pertinente señalar que para que la categoría HSH sea útil y válida dentro de un programa de prevención, deberá estar sustentada desde una perspectiva construccionista del género y la sexualidad y llenarse de contenidos socioculturales ale- jados de los discursos esencialistas. Para que las acciones y políticas públicas puedan hacerse cargo de estas nociones de sexualidad, deben dar cuenta de la naturaleza compleja de su objeto (ligado a prácticas subjetivas sociales) que escapa a las definiciones clásicas de la epidemiología y la salud pública y, por ende, tienen el reto de encontrar otras narrativas y discursos para poder llegar a mayor número de personas con mensajes que les sean relevantes $(13,38-40)$.

\section{Estudios en sexualidad masculina}

En América Latina son diversas y desiguales las publicaciones que dan cuenta del diagnóstico de la situación respecto a la sexualidad masculina y las relaciones sexuales entre hombres. A finales del siglo xx se abrieron espacios ligados a la globalización que propiciaron intercambios y encuentros entre los representantes de las organizaciones de base comunitaria, las personas que viven con VIH/SIDA y los y las activistas de organizaciones que trabajan en torno a las identidades sexuales. Estos intercambios fortalecieron la capacidad de respuesta y propuesta de estos grupos locales.

Los desarrollos académicos que surgieron en ese momento acompañaron las transformaciones sociales, políticas, económicas y culturales que se han dado en la región en las últimas décadas y han contribuido a una relativa democratización de las relaciones entre hombres y mujeres y al cuestionamiento de los discursos vigentes sobre masculinidad. Muchas de las instituciones que daban soporte al predominio masculino en el campo de la política, la economía y el control de la reproducción sufrieron cuestionamientos que repercutieron en las representaciones de género $\mathrm{y}$, en consecuencia, en la masculinidad. Así, ganaron amplio terreno en todo el mundo los estudios sobre las "mas- 
culinidades", tanto las hegemónicas como las alternativas, las no hegemónicas, las masculinidades femeninas y otra serie de propuestas de entendimiento de construcción de lo masculino $(29,40-42)$.

El discurso que sostenía la superioridad del hombre sobre la mujer ha perdido legitimidad. En Colombia, como en otros países latinoamericanos, el modelo de familia centrado en un varón proveedor ha sido reemplazado por un modelo familiar en el que participan indistintamente hombres y mujeres (42-46), y aunque no haya traído consigo cambios significativos en el comportamiento real de los varones y en la construcción de nuevos modelos de ser hombre, la ruta y la brecha para la transformación están allí para ser transitadas y exploradas.

Si bien las representaciones de la masculinidad están cambiando, en el ámbito de la sexualidad se sigue esperando que los hombres sean activos, tengan más de una pareja $\mathrm{y}$ tiendan a controlar la frecuencia y la forma en que mantienen relaciones sexuales. Muchos grupos sociales continúan propiciando que los hombres adopten actitudes frente al riesgo que los llevan a rechazar los preservativos por considerarlos "poco masculinos", a probar y a volverse adictos a las drogas inyectables y a ser negligentes con la prevención y el tratamiento de las infecciones de transmisión sexual. Esto incluye por descontado a la mayoría, sino a la totalidad de hombres sin distingo de su adscripción y orientación sexual, dado que se han construido total o en parte en este modelo de lo masculino que ha sido hegemónico (47).

Teniendo en cuenta la extensión del VIH, se plantea la necesidad de enseñar que el autocuidado y el cuidado de los otros también son cuestión de "hombres" y de ser masculinos. En un periodo de aumento de los índices de infección por viH, sobre todo entre los jóvenes, se hace indispensable trabajar para cambiar los patrones culturales que aseguran que "los hombres de verdad" son aquellos que corren riesgos, incluidos los que afectan su salud y su vida.

Muchos hombres y niños latinoamericanos continúan creyendo que es de "machos" tomar riesgos o incluso tener relaciones sexuales a la fuerza. Por otra parte, el uso de métodos anticonceptivos por parte de algunos varones no es necesariamente un sinónimo de previsión familiar y asunción de responsabilidades y puede, en ocasiones, ser un indicador de todo lo contrario: una huida de los compromisos y responsabilidades conyugales y familiares a través de la adopción de una actitud defensiva hacia la sexualidad protegida, que se asume, en términos peyorativos, como "femenina".

De Keijzer (48) vincula la socialización masculina con algunos comportamientos de violencia intrafamiliar, abuso y hostigamiento sexual, escasa participación en la anticoncepción y durante el embarazo, pero también con las principales causas de muerte masculina. Retomando la tríada de la violencia de la que habla Kaufman (49), este autor propone el concepto del varón como factor de riesgo para la salud, en tres ámbitos: las relaciones con las mujeres, las relaciones con los demás varones -en particular los que exhiben masculinidades no hegemónicas- y las relaciones consigo mismo, por medio del cual visibiliza los efectos nocivos para la salud que tiene la forma en que aprenden a interactuar los varones en el marco de una masculinidad hegemónica.

Queda de presente que ganar una comprensión más compleja de la sexualidad masculina exige estudiar no solo las prácticas sexuales de los varones, sino también los contextos sociales, culturales, económicos y políticos en los cuales se producen estas prácticas, los 
sistemas sociales y culturales en los cuales adquieren significados y las redes de poder en las cuales están imbricadas $(13,42,50)$.

En gran parte de América Latina, un hombre que penetra a otro hombre no deja de ser considerado viril (por el contrario confirma su hombría), pero un hombre que es penetrado casi siempre es estigmatizado como afeminado, y existe una tendencia a asociar la masculinidad con la heterosexualidad y la actividad y, de otro lado, la homosexualidad con la feminidad y la pasividad. Este esquema explica por qué muchos hombres declarados heterosexuales no admiten o no perciben haber tenido relaciones homosexuales. Su condición de penetradores les garantiza a sus ojos y socialmente su virilidad. Por otra parte, la discriminación y la hostilidad que existe en nuestra sociedad respecto a la homosexualidad los induce a ser reacios a admitir su comportamiento o prácticas homoeróticas. Y aunque el sexo entre hombres ocurre en todas las sociedades, las políticas gubernamentales y de salud no han tenido suficientemente en cuenta hasta el momento la necesidad de proteger a los HsH y a sus parejas, ya sean hombres o mujeres $(45,46,51,52)$.

La adopción de rasgos o comportamientos identificados como masculinos o femeninos, o de un rol pasivo o activo en las relaciones sexuales, es independiente de la orientación sexual. Las investigaciones destacan la complejidad de la relación entre papeles sexuales, papeles de género e identidad, y encuentran en ocasiones disidencias de la identidad, como es el caso de algunos HSH, en quienes la identidad no se genera solo a partir de elementos unívocos masculinos o femeninos. En ese sentido, se ha buscado mostrar que el comportamiento homosexual o heterosexual no está necesariamente asociado con un sentido diferenciado de identidad sexual.
Serrano (53) plantea que la "homosexualidad" es una categoría construida para referirse a ciertos aspectos de la vida de los seres humanos que rebasa los componentes sexuales e implica toda una expectativa de vida y una forma de entender y sentir el mundo. A través de sus prácticas, los homosexuales le otorgan nuevos significados a las categorías y papeles que la sociedad les impone. De esta manera, resuelven la tensión entre la identidad que se les propone socialmente y la identidad que ellos elaboran y recrean.

García (54) desarrolla un análisis sociolingüístico orientado a mostrar las relaciones entre el fenómeno de la prostitución masculina y los procesos de violencia y discriminación social. El autor analiza y muestra la heterogeneidad de situaciones que encubre la palabra homosexual y la diversidad de actores y sectores sociales que participan de esta forma de vida.

Otra situación interesante es aquella donde los hombres se encuentran confinados, sin posibilidades de intercambio afectivo o sexual con mujeres (cárceles, fuerzas armadas, órdenes religiosas), escenarios donde se configuran unas complejas experiencias homoeróticas que dificultan, particularmente, las acciones de prevención del viH/sIDA.

Durante toda la década de los noventa y en la primera década de los 2000, Parker se interesó por los problemas de salud sexual y reproductiva en relación con la formación de comunidades sexuales. El surgimiento (ante la difusión del VIH/SIDA) de nuevas comunidades homosexuales u homoeróticas, con sus propias estructuras institucionales y representaciones sociales, ha llamado la atención sobre la dinámica social y los procesos económicos y políticos que modelan la constitución de las comunidades sexuales, particularmente en los países en desarrollo, en un sistema mundial más amplio, con 
perspectivas de avanzar en el debate en América Latina y el resto de países del tercer mundo que aparecen bastante afectados por epidemias generalizadas en sus grupos de HSH $(47,50,55,56)$.

\section{Vulnerabilidad(es) de loS HSH}

Estudios recientes, que también utilizan metodologías cualitativas, han intentado ahondar en aquellos aspectos que llevan a que algunos o muchos de los hombres pertenecientes a la población HSH practiquen encuentros sexuales no protegidos, a pesar de que al ser entrevistados afirman conocer las consecuencias de este tipo de comportamiento (57-59).

Se ha llegado a la conclusión de que en esta compleja problemática influyen aspectos que van más allá de experiencias relacionadas solamente con las relaciones sexuales, como pueden ser la reafirmación de la autoestima y del sentimiento propio de ser atractivo para las demás personas, así como el peso que tienen los sentimientos de soledad emocional y la búsqueda de compensación de este hecho, la necesidad de conectar e intimar con otros $\mathrm{HSH}$, el hecho de sentirse enamorados, la visualización del riesgo como una fuente de placer y el deseo de rebelarse contra reglas establecidas.

Otras explicaciones de hombres entrevistados para no utilizar condón durante las relaciones sexuales entre hombres, incluyen la dificultad para utilizarlo (ligada a la pérdida de erección), el optimismo con respecto a los avances en el tratamiento de la infección por el VIH al estar disponibles mejores y más potentes antirretrovirales, actitudes de rechazo asociadas al cansancio de las campañas de prevención del VIH/SIDA y el consumo de alcohol y drogas, entre otras razones $(60,61)$.
Las conductas con respecto al sexo no protegido entre hombres se construyen sobre creencias, mensajes no verbales o supuestos personales basados en la intuición, en interpretaciones personales, en la confianza y estadios emocionales, es decir, los significados del sexo no protegido o de alto riesgo son tan variados como lo son las personas y cambian dependiendo de las circunstancias en las que se encuentren estos hombres $(13,59,62)$.

La sexualidad debe ser considerada una construcción social, una actividad dependiente de circunstancias sociales y culturales, un fenómeno que expresa una dimensión subjetiva, intersubjetiva y sociocultural (35). Como se dijo anteriormente, a pesar del grado de conocimiento de condiciones de vulnerabilidad y la información acerca del VIH que maneja la población HSH, las relaciones sexuales de alto riesgo se explican en la medida en que satisfacen ciertas necesidades no sexuales o psicológicas, como por ejemplo de tipo afectivo y socioemocional $(60,63)$.

Por ejemplo, la elección del compañero sexual en la población HSH depende de varios atributos, dentro de los que se incluyen el hecho de ser masculino (una actitud femenina expresada por un potencial compañero sexual lo convierte para muchos en pareja indeseable), la belleza, la juventud y el hecho de no estar sobrepesado, entre otros $(59,61)$.

El deseo de estar con una persona motivado por una fuerte atracción física puede llevar a los HSH a tener relaciones sexuales anales no protegidas. El acto sexual así considerado se convierte en un modo de afirmar la sensación de ser atractivo para los demás y de ser sexualmente deseable $(64,65)$. La necesidad de sentir pasión y la oportunidad de conectar con una pareja particularmente deseable, situación a veces facilitada por el consumo de alcohol y/o drogas, también puede llevar 
a encuentros sexuales no protegidos entre HSH. Debido al calor del momento, la situación se puede tornar complicada cuando los hombres que se sienten en desventaja por algún motivo (ya sea por edad, raza o por la creencia de carecer de atractivos físicos), temiendo ofender a la pareja deseable, acceden a prácticas sexuales no protegidas, inicialmente para no perder ni la oportunidad de interactuar sexualmente, ni la de alimentar su propia autoestima (61).

La búsqueda de compañeros sexuales ocasionales sirve para compensar sentimientos de vacío y soledad entre los HSH, sentimientos que inducen a estados de baja autoestima y a incurrir en situaciones de vulnerabilidad en el intento de conectar a través del sexo, lo que concuerda con la idea generalizada de que condiciones de depresión y poca autoestima podrían explicar la transmisión del VIH. Vivir y sentirse solo y estados emocionales negativos (particularmente el estrés y la depresión) se convierten en una plataforma potencial para verse involucrados en encuentros donde se producen relaciones anales no protegidas. En profundidad, detrás de la búsqueda de parejas sexuales ocasionales se esconden necesidades afectivas que se disfrazan como deseo sexual $(59,61)$.

Sumado a todo lo expresado hasta aquí, existe la creencia, la idea generalizada en la población HSH, de que el uso del condón impide la posibilidad de unirse totalmente con la otra persona. Podría interpretarse, entonces, que el hecho de practicar relaciones sexuales anales no protegidas con desconocidos busca fortalecer la unión íntima que se ha creado en ese momento con la otra persona, no solo en términos de placer físico, sino también como símbolo de unión emocional, de "enamoramiento", ya que sentirse enamorado del otro, aún sin compartir una relación estable o duradera con esa persona, conduce también a prácti- cas de penetración anal no protegida, como si se tratara de una demostración del sentimiento de amor, una muestra de intimidad que marca. En algunos casos, la transición hacia una relación afectiva más seria y que simboliza ese enamoramiento que se siente por el otro, lo cual puede conllevar contactos ocasionales que comprometan sexo no protegido, debido al amor y la confianza generada entre los dos $(59,66)$.

Algunos HsH podrían estar en la búsqueda de otro tipo de sensaciones, más allá del placer físico. Una búsqueda relacionada con la experimentación de sentimientos producidos al ponerse en una situación de riesgo que permita alcanzar un alto grado de excitación, asociado al hecho de haber cometido un acto prohibido: el riesgo se convierte entonces en un elemento de placer. Las relaciones sexuales no protegidas entre HsH podrían convertirse en un acto simbólico de rebelión y transgresión placentero, del cual probablemente no se está del todo consciente. Ese placer por lo prohibido, asociado al deseo de rebelarse contra normas establecidas, es una de las razones más comúnmente encontradas en el estudio de las motivaciones no sexuales para incurrir en situaciones de penetración anal no protegida entre HSH. Una conducta visualizada como vía de escape de la tensión producida por tener que vivir de acuerdo a las reglas $(63,66)$.

Opuesta a esta condición, debido a que las prohibiciones traen consigo sus propios deseos, se encuentra el sexo anal intencionalmente no protegido (conocido como barebacking), que para algunos individuos de la población HSH es la expresión máxima de la erotización del riesgo, el disfrute total de algo extremamente prohibido, a la vez asociado directamente con sentimientos autodestructivos y con la muerte $(59,67)$. 


\section{Conclusiones}

Con base en los estudios revisados se puede concluir que la relación entre comportamiento sexual e identidad de género es de una gran complejidad y que las formas a través de las cuales se construyen las identidades sexuales, en este caso "homosexuales", en distintos contextos, dependen en gran parte de las categorías y clasificaciones disponibles en cada sociedad y cultura para abordar la sexualidad. El foco de los estudios se ha desplazado del comportamiento sexual en sí mismo hacia las situaciones sociales en las cuales se produce dicho comportamiento y hacia las normas culturales que lo organizan. Desde este punto de vista, se han vuelto importantes las categorías "culturales locales" y los sistemas de clasificación que estructuran y definen la experiencia sexual en los distintos contextos sociales. Se ha hecho evidente que categorías como homosexualidad o heterosexualidad no reflejan la diversidad y complejidad de las experiencias sexuales vividas y se ha disociado el comportamiento homosexual o heterosexual de un sentido diferenciado de identidad de género.

Para muchos HSH, los mensajes de prevención pierden de vista la complejidad de las motivaciones de los hombres para tener relaciones sexuales, así como los sentidos emocionales que se asignan a estas. Los planteamientos del sexo protegido o del uso del condón pueden resultar ofensivos por los supuestos que connotan (de infidelidad, de tener infecciones de transmisión sexual, de desconfianza en la pareja) o por la complejidad de las interpretaciones que generan. El sexo no protegido se ve como facilitador de una mayor cercanía e intimidad con la pareja "estable" y se asumiría como una práctica normal, libre de riesgo.

54 Para estos hombres, el cálculo del riesgo de los compañeros sexuales se sigue haciendo a partir de su apariencia de persona "sana" o "enferma" y esto induciría a tener sexo sin protección con muchos de estos compañeros que lucen sanos. La baja autoestima, así como la clandestinidad de las experiencias sexuales (facilitadas por alcohol o drogas) a las que se enfrentan muchos hombres, afectan la capacidad cognitiva y emocional de los sujetos para adoptar conductas preventivas.

La dificultad de la incorporación del uso del condón es una realidad en muchos de los escenarios de la sexualidad homoerótica, en tanto en dichos escenarios otros riesgos estarían presentes y serían valorados como más importantes (como la integridad física, el riesgo de ser detenidos y encarcelados, el riesgo de perder la oportunidad del encuentro sexual, el sentirse señalado como persona enferma). Por lo anterior, se puede afirmar que no todos los HsH están listos para mensajes directos y contundentes, y por tanto muchos de ellos requieren mensajes o aproximaciones elípticas, y considero que una estrategia de intervención en HSH debería estar constituida por un discurso que promueva el respeto y la equidad de género, revalorice la sexualidad y permita la libre expresión y ejercicio de las diversas orientaciones sexuales. La construcción de comunidades gay y de espacios culturales que permitan el desarrollo de identidades homosexuales desde más temprano, contribuirá a que los HSH desarrollen mejores percepciones sobre el valor de su vida y por ende se esfuercen más en conservarla.

Las necesidades no sexuales de los HSH, que los llevan a comprometerse en situaciones de sexo no protegido, comprenden una gran variedad de situaciones, sensaciones y sentimientos que condicionan el modo de relacionarse con otros hombres. Por este motivo, los programas de prevención deben incluir y enfatizar los diferentes procesos, incluidos los afectivos, que intervienen en 
la toma de decisiones y en la aceptación de situaciones de vulnerabilidad para reorientar los mensajes dirigidos a la población HsH. De igual manera, es fundamental fortalecer la habilidad de los individuos HSH para formar y desarrollar relaciones sexuales y románticas con otros hombres sin descartar el riesgo potencial de transmisión del virus del VIH.

\section{Referencias bibliográficas}

1. ONUSIDA. Consulta regional sobre programas de prevención, atención y apoyo en VIH/SIDA con hombres que tienen sexo con hombres en América Latina y el Caribe. Ginebra: Autor; 1999.

2. ONUSIDA. Los hombres marcan la diferencia. Ginebra: Autor; 2001.

3. Guajardo G. Contexto sociocultural del sexo entre varones. En: Cáceres C, Pecheny M, Terto $\mathrm{V}$, editores. SIDA y sexo entre hombre en América Latina: Vulnerabilidades, fortalezas, y propuestas para la acción. Perspectivas y reflexiones desde la salud pública, las ciencias sociales y el activismo. Lima: Universidad Peruana Cayetano Heredia, Red de investigación en Sexualidades y vIH/SIDA en América Latina; 2002.

4. Cáceres C, Pecheny M, Terto V, editores. SIDA y sexo entre hombres en América Latina: Vulnerabilidades, fortalezas y propuestas para la acción. Perspectivas y reflexiones desde la salud pública, las ciencias sociales y el activismo. Lima: Universidad Peruana Cayetano Heredia, Red de Investigación en Sexualidades y VIH/SIDA en América Latina; 2002.

5. UNAIDS. 2008 Report on the global AIDS epidemic. Ginebra: Autor; 2008.

6. UNAIDS. 2008 unAIDs Annual report. Towards universal access. Ginebra: Autor; 2009.

7. García A, Noguer I, Cowgill K. El viH/sidA en países de América Latina. Los retos futuros. Publicación científica y técnica No. 597. Washington: Organización Panamericana de la Salud, Banco Mundial; 2004.

8. ONUSIDA. Infección por VIH y SIDA en Colombia. Estado del arte 2000-2005. Santafé de Bogotá: Pro-Offset Editorial Ltda.; 2006.

9. Baral S, Sifakis F, Cleghorn F, Beyrer C. Elevated risk for HIV infection among men who have sex with men in low -and middle- income countries 2000-2006: A systematic review. Plos Medicine. 2007; 4 (12): 1901-11.

10. Beyrer C. Hidden yet happening: the epidemics of sexually transmitted infections and HIV among men who have sex with men in developing countries. Sex Transm Inf. 2008; 84, 410-2.

11. Cáceres C, Konda K, Segura E, Lyerla R. Epidemiology of male same-sex behaviour and associated sexual health indicators in low and middle-income countries: 2003-2007 Estimates. Sex Transm Inf 2008; 84 (S1): i49-i56.

12. Stall R, Hart G. The continuing evolution of research on sexually transmited infections among men who have sex with men. Sex Transm Inf. 2008; 84, 407-9.

13. Estrada JH, Vargas L. Invisibles, excluidos y vulnerables: la infección VIH/SIDA entre hombres que tienen sexo con hombres (HSH). en: SIDA y sociedad. Bogotá: Ediciones Aurora; 2011.

14. Cáceres C, Konda K, Pecheny M, Chatterjee A, Lyerla R. Estimating the number of men who have sex with men in low and middle income countries. Sex Transm Inf. 2006; 82: iii3-iii9.

15. Heckathorn, D. Respondent-driven sampling: A new approach to the study of hidden populations. Social Problems. 1997; 44 (2): 174-99.

16. Mantecón A, Juan M, Calafat A, Becoña E, Román E. Respondent-driven sampling: un nuevo método de muestreo para el estudio de poblaciones visibles y ocultas. Adicciones. 2008; 20 (2): 161-70.

17. Broadhead R, Heckathorn D. AIDs prevention outreach among injection drug users: Agency problems and new approaches. Social Problems. 1994; 41, 473-95.

18. Johnston L, Khanam R, Reza M, Khan S, Banu $\mathrm{S}$, Alam S. The effectiveness of respondent driven sampling for recruiting males who have sex with males in Dhaka, Bangladesh. AIDs Behav. 2008; 12, 294-304.

19. Johnston L, Malekinejad M, Kendall C, Iuppa I, Rutherford G. Implementation challenges to using respondent driven sampling methodology for HIV biological and behavioral surveillance: field experiences in international settings. AIDS Behav. 2008; 12, S131-S141.

20. Malekinejad M, Johnston L, Kendall C, Franco L, Raven M, Rutherford G. Using Respondent-Driven Sampling Methodology for HIV biological and behavioral surveillance in international settings: A systematic review. AIDS Behav. 2008; 12: S105-S 130.

21. Ramírez-Valles J, Heckathorn, D, Vázquez R, Díaz R, Campbell R. From networks to populations: the development and application of respondent driven sampling among IDU and Latino gay men. AIDS and Behavior. 2005; 9: 387-402.

22. Ramírez-Valles J, Heckathorn D, Vázquez R, Díaz R, Campbell R. The fit between theory and data in Respondent Driven Sampling: Response to Heimer. AIDS and Behavior. 2005; 9 (4): 409-14.

23. Yeka W, Maibani-Michie G, Prybylski D, Colby D. Application of respondent driven sampling to collect baseline data on FSWS and MSM for HIV risk reduction interventions in two urban centres in Papua New Guinea. Journal of Urban Health: Bulletin of the New York Academy of Medicine. 2006; 83 (7).

24. Kendall C, Kerr L, Gondim R, Werneck G, Macena R, Kerr M. An empirical comparison of respondent-driven sampling, time location sampling, and snowball sampling for behavioral surveillance in men who have sex with men, Fortaleza, Brazil. AIDS Behav. 2008; 12: s97-s104. 
25. Prieto F. Veinte años del VIH en Colombia, 19832003. Datos de la vigilancia epidemiológica. Informe Quincenal Epidemiológico Nacional. 2003; 8 (22): 353-68.

26. Colombia, Ministerio de Salud. Plan estratégico de la respuesta nacional ante la epidemia del VIH/SIDA. Años 2000-2003. Santafé de Bogotá: Autor; 2004.

27. AMFAR. La iniciativa HSH. New York: AMFAR, The Foundation for AIDS Research; 2006.

28. Tolbert K, Morris K, Romero M. Los hombres y el proceso de decisión respecto del aborto: hacia una teoría de las relaciones de género y el aborto. Santafé de Bogotá: Universidad Externado de Colombia; 1994.

29. Sevilla E. Modelos analíticos de las ciencias sociales para prevención de infección por VIH. Reflexiones a partir de una experiencia de análisis de encuesta CAP. Ponencia para el Seminario Taller de Vigilancia Epidemiológica e Investigación en VIH/sIDA. Santafé de Bogotá: Ministerio de Salud/unaids. Mecanografiado; 1999.

30. Manzelli H, Pecheny M. Prevención del VIH/SIDA en hombres que tienen sexo con hombres. En: Cáceres C, Pecheny M, Terto V, editores. SIDA y sexo entre hombre en América Latina: vulnerabilidades, fortalezas, y propuestas para la acción. Perspectivas y reflexiones desde la salud pública, las ciencias sociales y el activismo. Lima: Universidad Peruana Cayetano Heredia, Red de Investigación en Sexualidades y VIH/SIDA en América Latina; 2002.

31. Estrada JH. Masculinidades homoeróticas y la infección VIH entre hombres que tienen sexo con hombres. Culture, Health \& Sexuality. 2007; 9 (S1): S-85-S-86.

32. Estrada JH. Evaluación de programas educativos para jóvenes, como herramientas de prevención de VIH/SIDA. Revista Gerencia y Políticas de Salud. 2009; 8 (16): 72-90.

33. Family Health International, Impact, UNAIDS, USAID, World Health Organization, UNDCP. Estimating the size of populations at risk for HIV. Durham: Family Health International. 2003 [acceso: 16 de octubre de 2009]. Disponible en: http://www.fhi.org/NR/ rdonlyres/e66sj52tyha $7 \mathrm{~m} 5$ dozchbbwptxepfqr47vmvjxvyo2dy7trd2ne5giddtvkksddwrpwxatdgkprxwb a/EstimatingSizePop.pdf

34. Estrada JH. Modelos de prevención en la lucha contra el viH/sIDA. Acta Bioéthica. 2006; XII (1): 91-100.

35. Cáceres C. HIV among gay and other men who have sex with men in Latin America and the Caribbean: ¿a hidden epidemic? AIDS. 2002; 16 (Suppl.): S-23-S-33.

36. Choi K, Lui H, Guo Y, Han L, Mandel J. Lack of HIV testing and awareness of HIV infection among men who have sex with men, Beijing, China. AIDS Education and Prevention. 2006; 18 (1): 33-43.

37. Gomensoro A, Lutz E, Güida C, Corsino D. La nueva condición del varón. Montevideo: Editorial Fin de Siglo; 1995.

38. Cáceres C. Bisexualidades masculinas en la Lima de los noventa: consideraciones de salud sexual. En: El amor y sus especies. Lima: Pontificia Uni- versidad Católica del Perú, Facultad de Ciencias Sociales; 1995.

39. Cáceres C. Male bisexuality in Perú and the prevention of AIDS. En: Aggleton P, editor. Bisexualities and AIDS - International perspectives. Londres: Taylor \& Francis; 1996.

40. Leñero L. Los jóvenes varones solteros y sus familias. Revista Iztapala. 1992; 27: 199-218.

41. Calvez M. Los usos sociales del riesgo. Elementos de análisis cultural del sIDA. San Martín: Editorial Universitaria; 1998.

42. Viveros M, Estrada JH, Domínguez ME et ál. Masculinidad, homosexualidad, vIH-SIDA e identidad de género. Propuesta de intervención para hombres que tienen sexo con hombres. Bogotá: Ministerio de la Protección Social; 2010.

43. Fernández-Kelly P. Recasting women in the global economy. New York: The Russell Sage Foundation; 1993.

44. Fuller N. Dilemas de la femineidad: Mujeres de clase media en el Perú. Lima: Universidad Católica del Perú; 1993.

45. Viveros M. Contemporary Latin American Perspectives on Masculinity. Men and Masculinities. 2001; 3 (3): 237-60.

46. Viveros M. De quebradores y cumplidores. Santafé de Bogotá: CEs/Universidad Nacional de Colombia/ Profamilia/Fundación Ford; 2003.

47. Parker R. Estado de la investigación en sexualidad: Avances y desafíos sociales. Ponencia presentada en el I Seminario Taller Sudamericano en Investigación Sociocultural en Sexualidad. Prioridades y desafíos, Santiago de Chile.

48. De Keijzer B. Masculinity as a risk factor. Seminar on fertility and the male life cycle in the era of fertility decline. Zacatecas: IUSSP; 1995.

49. Kaufman M. Hombres: Placer, poder y cambio. Santo Domingo: Cipaf; 1989.

50. Parker R. Bisexuality and HIV/AIDs in Brazil. En: Aggleton P, editor. Bisexualities and AIDS - International perspectives. Londres: Taylor and Francis; 1996.

51. ONUSIDA. Vive y deja vivir. Ginebra: Autor; 2002.

52. OnUSIDA. Situación de la epidemia de SIDA. Ginebra: Autor; 2006.

53. Serrano J. Diversidad cultural y homosexualidades. Ponencia presentada en el Simposio Sexualidad y Construcción de Identidad de género, VII Congreso de Antropología en Colombia. Medellín: Universidad de Antioquia; 1994.

54. García C. Los pirobos del Terraza: interacción y discriminación sociales en un grupo de trabajadores sexuales. Monografía presentada como requisito para obtener el título de Licenciado en Filología e Idiomas, Facultad de Ciencias Humanas. Bogotá: Universidad Nacional de Colombia; 1994.

55. Parker R. Hacia una economía política del cuerpo: construcción de la masculinidad y la homosexualidad masculina en Brasil. En Valdés T, Olavarría J, editores. Masculinidades y equidad de género en América Latina. Santiago de Chile: Editorial Flacso, FNUAP; 1998. 
56. Parker R. Administering the Epidemic: HIV/AIDS, Policy, Models of Development and International Health. En Manderson L, Whiteford, L. Global health policy, local realities: The fallacy of the level playing field. Londres: Boulder; 2000.

57. Dodds J, Mercey D, Parry J, Johnson A. Increasing risk behaviour and high levels of undiagnosed HIV infection in a community sample of homosexual men. Sex Transm Inf. 2004; 80, 236-40.

58. Folch C, Casabona J, Muñoz R, Zaragoza K. Evolución de la prevalencia de infección por el viH y de las conductas de riesgo en varones homo/bisexuales. Gac Sanit. 2005; 19 (4): 294-301.

59. Fernández-Dávila P. The non-sexual needs of men that motivate them to engage in high-risk sexual practices with other men. Forum: Qualitative Social Research. 2009; 10 (2). [Acceso: 22 de octubre de 2009]. Disponible en: http://nbn-resolving.de/ urn:nbn:de:0114-fqs0902219

60. Adam B, Sears A, Shellenberg G. Accounting for unsafe sex: Interviews with men who have sex with men. The Journal of Sex Research. 2000; 37 (1): 24-36.

61. Adam B, Husbands W, Murray J, Maxwell J. AIDS optimism, condom fatigue, or self-esteem? Explain- ing unsafe sex among gay and bisexual men. The Journal of Sex Research. 2005; 42 (3): 238-48.

62. Fernández-Dávila P. "Amigos con derecho a roce": una oportunidad para contraer la infección por el virus de la inmunodeficiencia humana en hombres homo/ bisexuales con prácticas sexuales de alto riesgo. Gac Sanit. 2007; 21 (6): 471-78.

63. Perloff R. Persuading people to have safer sex: Applications of social science to the AIDS crisis. Mahwah: Lawrence Erlbaum Associates; 2001.

64. Instituto de Salud Pública (ISP), Comunidad de Madrid. Factores socioculturales relacionados con la realización de la prueba de detección de anticuerpos frente al $\mathrm{VIH}, \mathrm{y}$ con conductas sexuales de riesgo en el colectivo de varones que tienen relaciones sexuales con otros varones. Madrid: ISP, Comunidad de Madrid; 2004.

65. Belza M. Risk of HIV infection among male sex workers in Spain. Sex Transm Inf. 2005; 81, 85-8.

66. Crossley M. Making sense of "barebacking": Gay men's narratives, unsafe sex and the "resistence habitus". British Journal of Social Psychology. 2004; 43: 225-44.

67. Wolitski R, Valdiserri R, Denning P, Levine W. Are we headed for a resurgence of the hiv epidemic among men who have sex with men? American Journal of Public Health. 2001; 91(6): 883-8. 


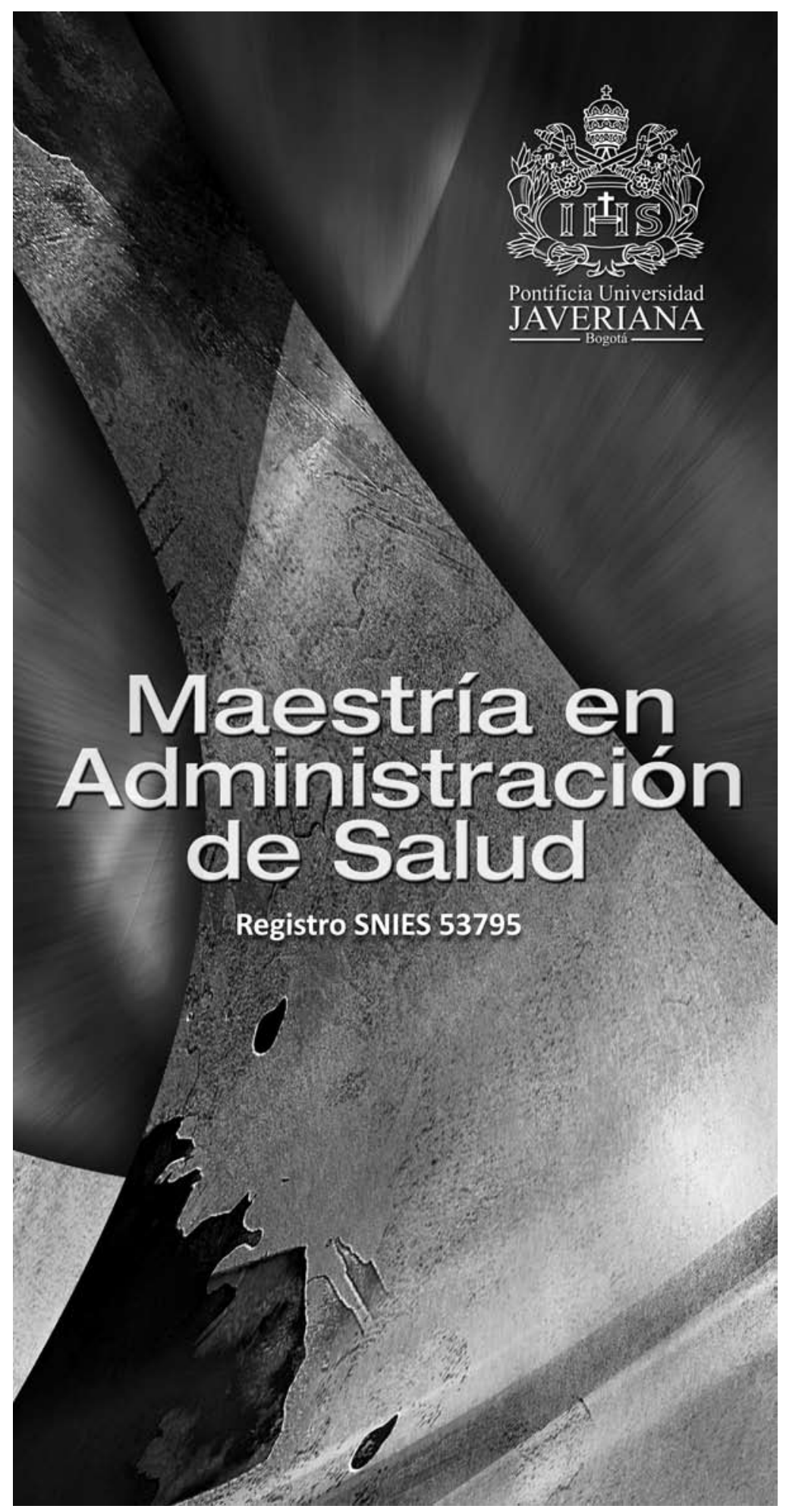

